\title{
SEVERAL NEW BEAM MEASUREMENT SYSTEMS FOR HLS*
}

\author{
$\underline{\text { B. G. Sun }}^{\dagger}$, J. H. Wang, P. Lu, D. H. He, H. L. Xu, J. Y. Li, J. H. Liu \\ NSRL, University of Science and Technology of China, Hefei, Anhui 230029, P.R.China
}

\section{Abstract}

This paper describes several new beam instruments for Hefei light source (HLS). These new beam instruments include a tune measurement system to measure the beam tune and chromaticity, a DCCT measurement system to measure the beam DC current and beam life, and a closed orbit measuring system to measure the beam position. Some applications of these measurement systems and their measurement results are presented.

\section{INTRODUCTION}

HLS is an $800 \mathrm{MeV}$ electron storage ring. To measure the beam parameters and help to understand the machine behaviour during the commissioning phase and operation, the beam diagnostic system was built $[1,2]$.

In Phase II of NSRL, the improvement of beam measurement system is one of important subjects. Based on this background, the several new beam measurement systems were developed for HLS.

\section{TUNE MEASUREMENT SYSTEM}

\subsection{Combination of tune measurement system}

The swept frequency excitation method was used to measure betatron tune. The tune measurement system [3] is shown as Fig.1.

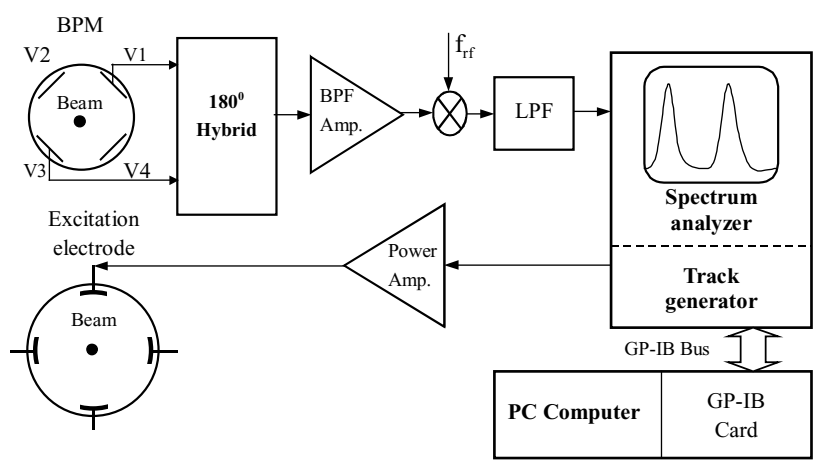

Figure 1: Block diagram of tune measurement system

The different signal from the stripline electrodes is demodulated by an AM detector. The signal will be analyzed by a spectrum analyzer with tracking generator. The output signal of the tracking generator is amplified by a power amplifier. The excitation electrode is driven by the amplified tracking signal to excite betatron oscillation.

\footnotetext{
* Supported by National Important Project on Science- Phase II of NSRL

†bgsun@ustc.edu.cn
}

\subsection{Measurement result of betetron tune}

Fig.2 shows the typical spectrum analyzer plots, in which the point 1 shows the frequencies of the fraction horizontal tune and the point 2 shows the frequencies of the fraction vertical tune. We may get that $v_{x}$ and $v_{y}$ is respectively 3.5482 and 2.6072 . The measurement accuracy is about $1 \times 10^{-4}$.

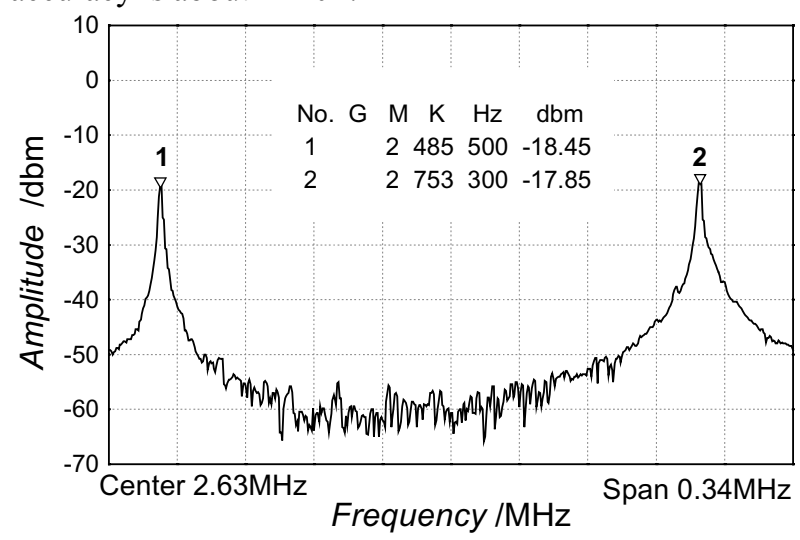

Figure 2: The typical tune signal

\subsection{Application of tune measurement system}

In HLS, the natural chromaticity and the corrected chromaticity ware measured by the tune measurement system.

The natural chromaticity can be obtained by detecting the variation of the tune as a function of the main dipole field strength. Fig. 3 shows the variation of the tune as a function of the main dipole field strength.

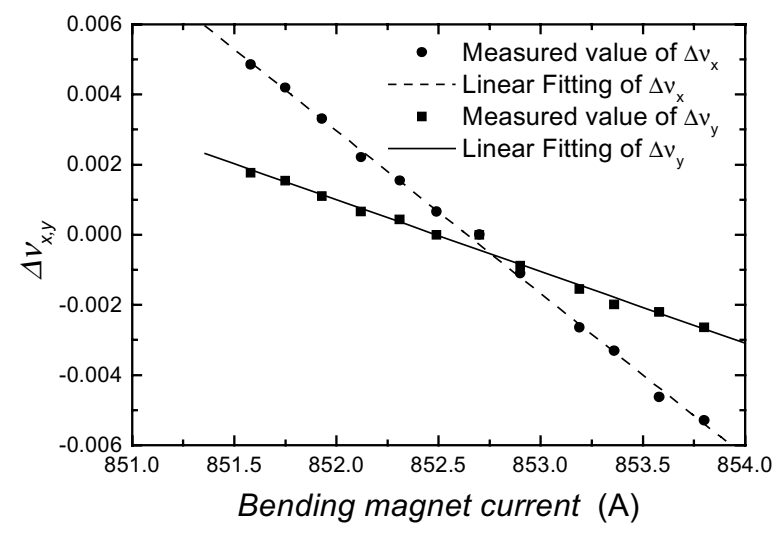

Figure 3: Tune variation as a function of the change in bending magnet current 
Here, the natural chromaticity in horizontal and vertical is respectively -6.059 and -2.634 .

The corrected chromaticity can be determined by measuring the tune shift as a function of the RF frequency. Fig.4 shows the variation of the tune as a function of the RF frequency change. Here, the corrected chromaticity in horizontal and vertical is respectively 0.268 and 3.057.

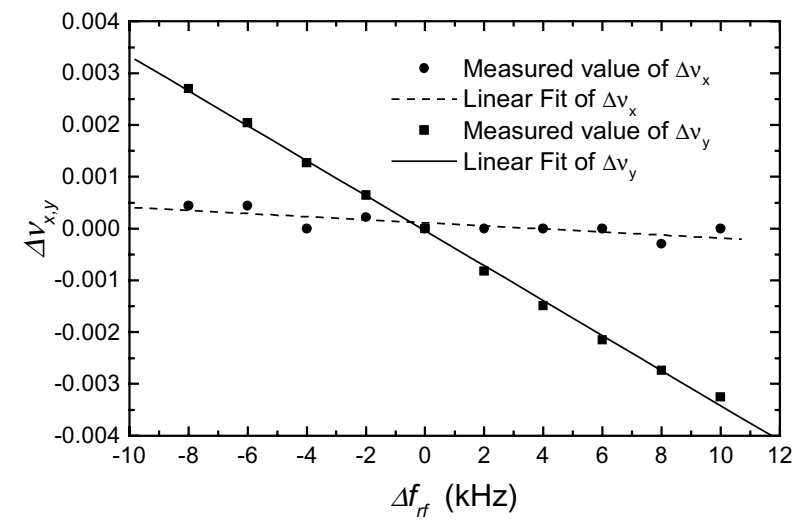

Figure 4: Tune variation as a function of the change in RF frequency

\section{DCCT MEASUREMENT SYSTEM}

\subsection{Combination of DCCT measurement system}

The new DCCT measurement system is developed for HLS. The system is composed a PCT system, a DCCT shielding system and a DCCT data acquisition system. The block diagram of the DCCT measurement system is shown as Fig. 5 .

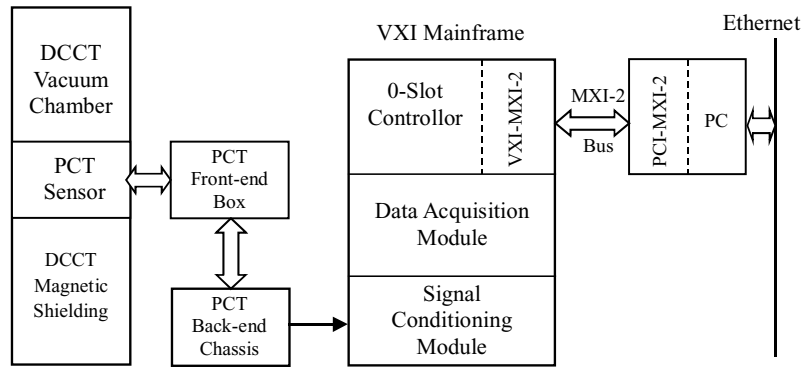

Figure 5: Block diagram of DCCT measurement system

The PCT unit was manufactured by Bergoz Co., France.

The mechanical design of the DCCT vacuum chamber and its magnetic shielding is shown as Fig.6. Here, two concentric cylinders ware applied so to reduce the external field affecting the PCT toroid. The transverse shielding factor of the two-layer arrangement is about $1 \times 10^{2}$. Because the injection energy is $200 \mathrm{MeV}$ and operation energy is $800 \mathrm{MeV}$, the ramping of a stored beam is accompanied by variations of stray fields from bending and quadrupole magnets. The influence of the stray fields on DCCT system has analyzed and measured [4]. Under no magnetic shielding, zero drifts of DCCT are about $1 \mathrm{~mA}$ during ramping process. Therefore, the design of the magnetic shielding of DCCT system is very necessary.

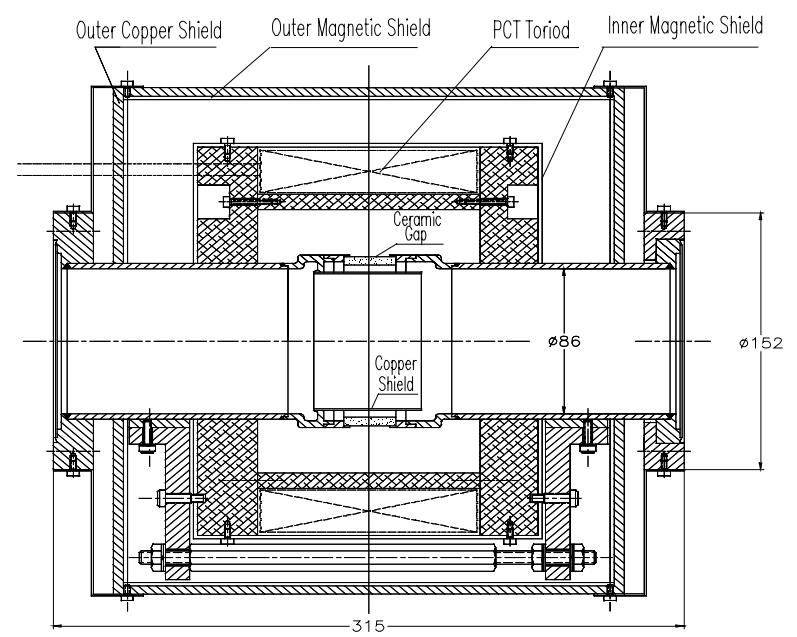

Figure 6: Mechanical design of the DCCT vacuum chamber and its magnetic shielding

The mechanism of affecting DC current transformer (DCCT) is analyzed under single bunch operation. The method of increasing ceramic gap capacitance is used, which ensure DCCT operate well under single bunch operation [5].

The DCCT data acquisition system consists of a VXI mainframe VX1410A, a 0-slit controller VXIPCI8015, a data acquisition module VXI-MIO-64XE-10, and a signal conditioning module VXI-SC-1102B. The DCCT acquisition software was written with LabVIEW.

\subsection{Measurement results of DCCT system}

The DCCT measurement system has been installed. In no beam, its specifications were measured, the linearity error $< \pm 0.01 \%$, the accuracy is about $0.1 \%$, the magnetic shielding against stray fields suppressed zero drifts of DCCT from $1 \mathrm{~mA}$ to $10 \mu \mathrm{A}$. Fig.7 shows the measurement result of the DC current using the DCCT measurement system under operation.

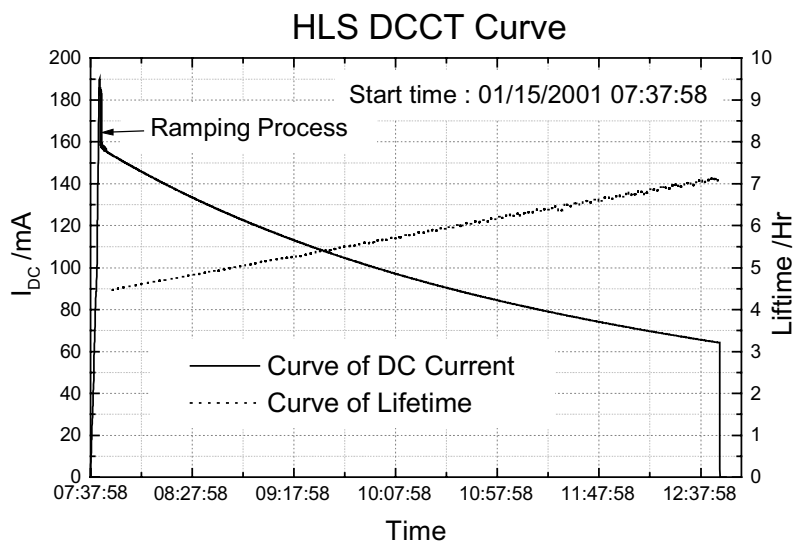

Figure 7: Curve of DC current using DCCT measurement system 


\section{CLOSED ORBIT MEASURING SYSTEM}

\subsection{Combination of closed orbit measuring system}

The new closed orbit measuring system is developed for HLS [6]. The system is consists of a total number of 22 BPMs, a set of BPM-electrons modules produced by French company Bergoz, and a VXI data acquisition system. The closed orbit acquisition software was written with LabVIEW. The block diagram of closed orbit measuring system is shown as Fig.8.

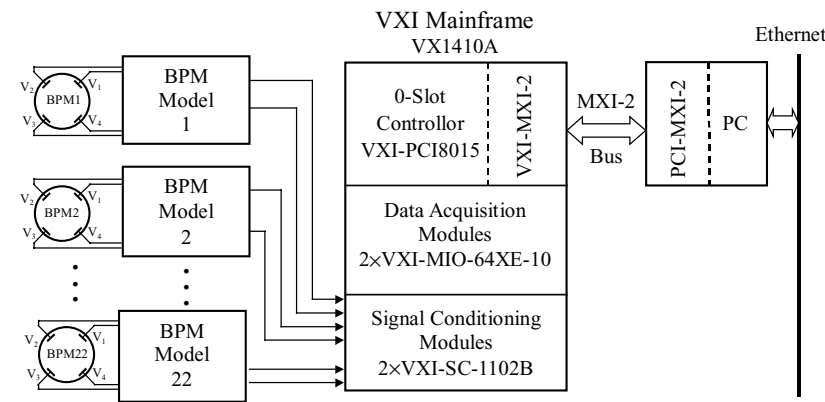

Figure 8: Block diagram of the closed orbit measuring system

\subsection{Application of closed orbit measuring system}

In HLS, the dispersion was measured by the closed orbit measuring system. In electron storage ring, the dispersion is inferred from the orbit change induced by a shift in the RF frequency, measuring the orbit change with BPM system. Comparing the dispersion measured by new closed orbit measuring system with the theoretic dispersion is shown in Fig.9

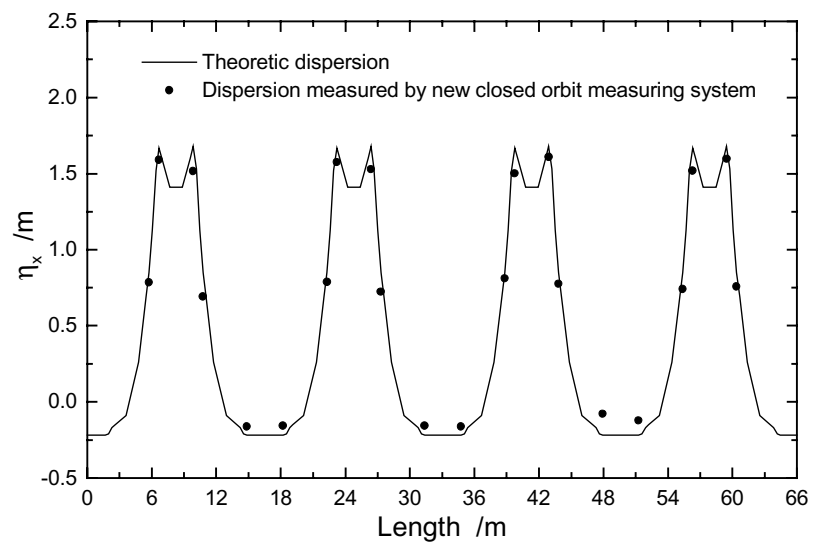

Figure 9: Comparing the measurement dispersion with the theoretic dispersion

\subsection{Results of the initial beam-based} calibration of closed orbit measuring system

In HLS, the offset of the beam position monitor (BPM) with respect to the magnetic center of a quadrupole was measured using method of the beam- based alignment [7]. A switchable shunt resistor was installed on quadrupole to bypass a small percentage of the magnet current, and solid-state relays was used to switch the shunt resistor.

Fig. 10 shows an example of the experimental result in vertical for Q7W. Here, when the beam was passing through the center of the quadrupole Q7W, the BPM17 read $-1.81 \mathrm{~mm}$ vertically.

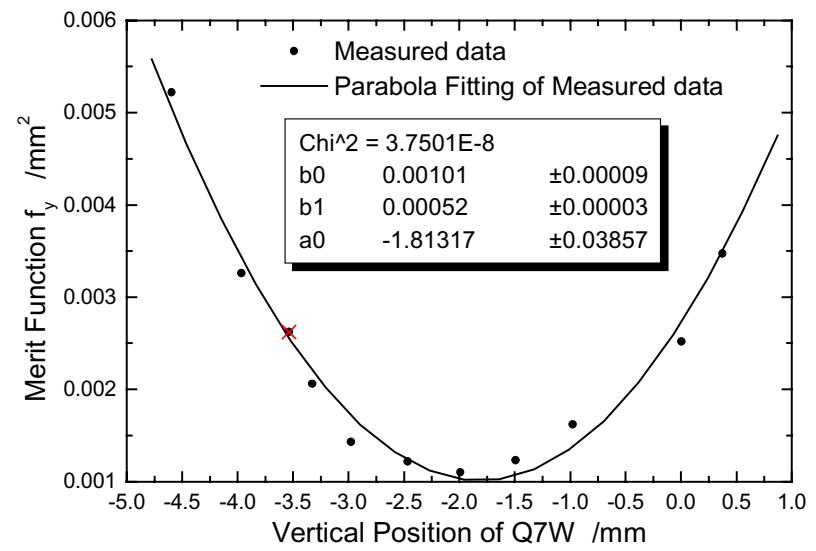

Figure 10: An example of the beam-based calibration in vertical for Q7W

\section{CONCLUSION}

Several new beam instruments for HLS have performed very well thus far. They play a crucial role in pushing to ultimate machine performance and in doing advanced machine physics.

\section{REFERENCES}

[1]Y. Yin. "The Beam Diagnostics for Hefei SR Machine". Proceedings of the International Conference on Synchrotron Radiation Applications, 1989, pp.265269.

[2]B. G. Sun, P. Lu, et al. "Beam measurement system in NSRL". Journal of System Engineering and Electronics, Vol.11, No.3, 2000, pp.130-135

[3]B. G. Sun, D. H. He, et al. "Tune Measurement System for HLS". Nuclear Techniques, Vol. 24, No. 1, 2001.1, pp47-51

[4]B. G. Sun, D. H. He, et al. "Magnetic Shielding of DCCT System for HLS". High Power Laser and Particle Beams, Vol. 12, No. 4, Aug. 2000, pp.525-527

[5]B. G. Sun, D. H. He, et al. "Mechanism analysis and improvement measurement of affecting DCCT under single bunch operation". Atomic Energy Science and Technology, Vol.33, No.5, Sep. 1999, pp.404-409

[6]J. H. Wang, Z. P. Liu, B. G. Sun, et al. "The Updated BPM System of HLS". These proceedings.

[7] B. G. Sun. Thesis for Ph.D Degree. Published by University of Science and Technology of China, 2000 